\title{
Three-dimensional simulation of harmonic up-conversion in a prebunched two-beam free-electron laser
}

\author{
M. H. Rouhani* and B. Maraghechi \\ Department of Physics, Amirkabir University of Technology, P.O. Box 15875-4413, Tehran, Iran
}

(Received 31 August 2009; published 18 August 2010)

\begin{abstract}
Three-dimensional simulation of harmonic up-conversion in a free-electron laser amplifier operating simultaneously with two cold and relativistic electron beams with different energy is presented in the steady-state regime. The fundamental resonance of the higher energy beam is adjusted at the third harmonic of the lower energy beam. By using slowly varying envelope approximation, the hyperbolic wave equations can be transformed into parabolic diffusion equations. By applying the source-dependent expansion to these equations, electromagnetic fields are represented in terms of the Hermite Gaussian modes which are well suited for the planar wiggler configuration. The electron dynamics is described by the fully three-dimensional Lorentz force equation in the presence of the realistic planar magnetostatic wiggler and electromagnetic fields. A set of coupled nonlinear first-order differential equations is derived and solved numerically. This set of equations describes self-consistently the longitudinal spatial dependence of radiation waists, curvatures, and amplitudes together with the evaluation of the electron beam. The evolutions of the transverse modes, in this system, are investigated for the fundamental resonance and its harmonic up-conversion. In addition to uniform beam, prebunched electron beam has also been studied. The effect of sinusoidal distribution of entry times for low energy electron beam on the evolution of radiation is compared with water bag distribution. It is shown that prebunching reduces the saturation length substantially. The analysis is related to extreme ultraviolet and x-ray emission where by seeding the lower frequency of the fundamental resonance of the lower energy beam substantial power is obtained at its third harmonic.
\end{abstract}

DOI: 10.1103/PhysRevSTAB.13.080706

PACS numbers: 41.60.Cr, 42.60.Jf, 42.65.Ky, 02.60.Cb

\section{INTRODUCTION}

The main concern in free-electron laser (FEL), nowadays, is the production of coherent high power short wavelength radiation because it has the potential to open new regimes in atomic and electronic processes [1-5]. The main problem in this field is to find processes or phenomena for seeding the FEL. In one method, the stochastic bunching in the electron beam, due to shot noise, was exploited [6]. Since the self- amplified spontaneous emission (SASE) starts from electron beam shot noise, the output of the system typically has limited temporal coherence and relatively large shot-to-shot fluctuations in both power and spectrum although it has transverse coherence [7].

In order to improve the longitudinal coherence, other methods have been proposed such as injection of harmonics, which is generated in gas [8], classical or nonclassical high-gain harmonic generation [9-11], and two-beam FEL for frequency up-conversion [12]. In these methods harmonic bunching play an essential role. Radiation of the electron beam in the planar undulator contains a rich harmonic spectrum. Higher harmonic radiation can significantly extend the operating band of user facilities.

\footnotetext{
*mohammad_hadi_rouhani@yahoo.com
}

In the two-beam frequency up-conversion method which was proposed by McNeil et al. [12], two relativistic electron beams with different energies were exploited in a FEL. The higher energy electron beam is chosen so that its fundamental resonance wavelength is a harmonic resonance wavelength of the lower energy beam. It is shown in Ref. [12] that it is possible to seed the copropagating electron beams, externally, at the fundamental resonance of the lower energy electron beam. The lower energy beam will be bunched at its fundamental resonance as well as at its harmonics and will retain the coherence properties of the seed. This process is strongly coupled with the higher energy beam because its fundamental FEL interaction is at one of the harmonics of the lower energy beam. This coupling between the lower and higher frequency FEL interactions allows the coherence properties of the longer wavelength seed field to be transformed to the unseeded short harmonic wavelength interaction.

This phenomenon has not been studied in three dimensions neither in the averaged form nor in the nonaveraged method. Therefore, three-dimensional features such as diffraction, radiation guiding, and evolution of transverse modes have not been considered in this system. The optical guiding of light in FEL [13-15] results during amplification when the coherent interaction between the source electron beam and the electromagnetic field introduces 
an inward curvature in the phase front of the light, which refracts it back toward the lasing core of the electron beam. During the amplification the electron beam can behave like a guiding structure that suppresses diffraction, reduces transverse power losses, and enhances the electromagnetic field amplification. The evolution of transverse modes is important in planning for future user facilities that intend to employ radiation from this system [16]. Although the multiple electron beam FEL was studied in [17], in three dimensions, the harmonic up-conversion was not considered. The main interest in that paper was to reduce the coherent synchrotron radiation in the bunch compressor by adding energy spread to the electron beam.

The purpose of the present study is to present a threedimensional nonaveraged simulation of two-beam FEL by using source-dependent expansion (SDE) [18-22]. The novel aspect of the SDE method is that the characteristics of the modes are governed by the driving current density. Therefore, instead of using the usual modal expansion consisting of vacuum Laguerre-Gaussian or HermiteGaussian functions [23], the source function is, in SDE, incorporated self-consistently into the functional dependence of the radiation waist, the radiation wave front curvature, and the radiation amplitude. The effect of prebunching of low energy electron beam on the evolution of radiation in the system is studied. Using low energy prebunched electron beam saturation length can be reduced dramatically.

It is important to emphasize that no average is performed over the Lorentz force equation; therefore the KrollMorton-Rosenbluth (KMR) scheme is not used. In the KMR method [24-29], the electron trajectories are averaged over the wiggler period. Hence, only two equations are integrated per electron, specifically, for the energy and ponderomotive phase. Advantages of the non-KMR approach are the possibility to treat the injection of the beam into the wiggler, the ease of inclusion of external focusing or dispersive magnetic components in the beam line, and the facility for using an actual magnetic field in the numerical solution.

The code which is written for this purpose is named modified MEDUSA code. Because we did not have access to the original MEDUSA code [30], we first rewrote the code and then modified it to study the two-beam FEL.

The organization of the paper is as follows. The general formulation is described in Sec. II, including the wiggler model, the dynamical field equations with SDE, and the Lorentz force equations. The simulation is preformed to study the two-beam FEL in Sec. III. Section IV is devoted to the summary and suggestions.

\section{BASIC EQUATION}

The tapered planar wiggler magnetic field, produced by magnets with parabolically shaped pole faces, may be described by

$$
\begin{aligned}
\mathbf{B}_{w}(\mathbf{x})= & B_{w}(z)\left\{\operatorname { c o s } ( k _ { w } z ) \left[\hat{\mathbf{e}}_{x} \sinh \left(\frac{k_{w} x}{\sqrt{2}}\right) \sinh \left(\frac{k_{w} y}{\sqrt{2}}\right)\right.\right. \\
& \left.+\hat{\mathbf{e}}_{y} \cosh \left(\frac{k_{w} x}{\sqrt{2}}\right) \cosh \left(\frac{k_{w} y}{\sqrt{2}}\right)\right] \\
& \left.-\sqrt{2} \hat{\mathbf{e}}_{z} \sin \left(k_{w} z\right) \cosh \left(\frac{k_{w} x}{\sqrt{2}}\right) \sinh \left(\frac{k_{w} y}{\sqrt{2}}\right)\right\}, \\
B_{w}(z)= & \begin{cases}B_{w} \sin ^{2}\left(\frac{k_{w} z}{4 N_{w}}\right) & 0 \leq z \leq N_{w} \lambda_{w} \\
B_{w} & N_{w} \lambda_{w}<z,\end{cases}
\end{aligned}
$$

where $B_{w}$ denotes the wiggler amplitude, $k_{w}=2 \pi / \lambda_{w}$ is the wiggler wave number corresponding to the wiggler period $\lambda_{w}$, and $N_{w}$ is the number of entry taper of wiggler periods. This type of wiggler provides enhanced focusing of the electron beam in the plane transverse to the direction of bulk electron flow. At the entry region, the wiggler amplitude varies with axial position in order to model the adiabatic injection of the beam into the wiggler.

The vector potential of the linearly polarized radiation field is

$$
\begin{gathered}
\mathbf{A}_{r}(\mathbf{x}, t)=\sum_{h} \mathbf{A}_{r, h}(\mathbf{x}, t), \\
\mathbf{A}_{r, h}(\mathbf{x}, t)=\frac{A_{r, h}(\mathbf{x})}{2} e^{i h(k z-\omega t)} \hat{\mathbf{e}}_{x}+\text { c.c. },
\end{gathered}
$$

where $A_{r, h}$ is the slowly varying complex radiation field amplitude, $\omega$ is the fundamental frequency, and c.c. denotes the complex conjugate. The wave equation is [20]

$$
\left(\nabla_{\perp}^{2}+\frac{\partial^{2}}{\partial z^{2}}-\frac{1}{c^{2}} \frac{\partial^{2}}{\partial t^{2}}\right) \mathbf{A}_{r}=-\frac{4 \pi}{c} J_{x} \hat{\mathbf{e}}_{x}
$$

where $J_{x}$ is the driving current density. Since the wavelength of interest is less than a micrometer, the collective Raman effects due to the beam space charge wave are neglected.

Substituting Eq. (2) into Eq. (4), using slowly varying envelope approximation, and averaging over one wave period, transforms the normally hyperbolic equation into a parabolic diffusion equation for harmonic amplitudes:

$$
\left(\nabla_{\perp}^{2}+2 i h k \frac{\partial}{\partial z}\right) A_{r, h}=-\frac{8 \pi}{c} \frac{\omega}{2 \pi} \int_{0}^{2 \pi / \omega} J_{x} e^{i h(k z-\omega t)} d t .
$$

The details of the derivation of Eq. (5) are given in the Appendix. The source-dependent expansion technique is applied to the above equation. Since the planar wiggler is used, the Hermite-Gaussian modal expansion will be employed. It is worth mentioning that for the system with circularly symmetric structure the Laguerre-Gaussian modes are better suited. For this reason the radiation amplitude represented in terms of Gauss-Hermite modes as 


$$
A_{r, h}=\sum_{l, n} e_{l, n, h}(x, y) e^{i \alpha_{h} r^{2} / w_{h}^{2}} \delta A_{l, n, h}
$$

where the summation indices $l$ and $n$ denote the transverse mode structure, $h$ is the harmonic number, and

$$
e_{l, n, h}(x, y)=e^{-r^{2} / w_{h}^{2}} H_{l}\left(\frac{\sqrt{2} x}{w_{h}}\right) H_{n}\left(\frac{\sqrt{2} y}{w_{h}}\right)
$$

describes the transverse structure of each mode. Here, $H_{l}$ is the Hermite polynomial of order $l, w_{h}$ is the spot size of the $h$ th harmonic, $\alpha_{h}$ is related to the curvature of the phase front, and $\delta A_{l, n, h}=\delta A_{l, n, h}^{(1)}-i \delta A_{l, n, h}^{(2)}$. It is assumed that $\delta A_{l, n, h}^{(1)}, \delta A_{l, n, h}^{(2)}, \alpha_{h}$, and $w_{h}$ are slowly varying functions in $z$.

To find the dynamical equations, substitute the mode representation, i.e., Eq. (6) into the parabolic diffusion equation (5). Then, by multiplying the result with $\exp \left(-i \alpha_{h} r^{2} / w_{h}^{2}\right)$ and orthogonalizing in $x$ and $y$ with the use of the following relation,

$$
\int_{-\infty}^{+\infty} e_{l, n, h}(x, y) e_{l^{\prime}, n^{\prime}, h}(x, y) d x d y=\frac{w_{h}^{2}}{2} \pi 2^{l+n} l ! n ! \delta_{l, l^{\prime}} \delta_{n, n^{\prime}} .
$$

The dynamical equation will be obtained,

$$
\begin{aligned}
& \left(\frac{d}{d \bar{z}}+\frac{1}{\bar{w}_{h}} \frac{d \bar{w}_{h}}{d \bar{z}}\right)\left(\begin{array}{c}
\delta a_{l, n, h}^{(1)} \\
\delta a_{l, n, h}^{(2)}
\end{array}\right)+\bar{K}_{l, n, h}\left(\begin{array}{c}
-\delta a_{l, n, h}^{(2)} \\
+\delta a_{l, n, h}^{(1)}
\end{array}\right) \\
& =\left(\begin{array}{c}
\bar{s}_{l, n, h}^{s,(1)}+\delta_{h, h^{\prime}} \bar{s}_{l, n, 1}^{f,(1)} \\
\bar{s}_{l, n, h}^{s,(2)}+\delta_{h, h^{\prime}} \bar{s}_{l, n, 1}^{f,(2)}
\end{array}\right),
\end{aligned}
$$

where $h^{\prime}$ is the highest harmonic for the low energy electron beam (superscript $s$ ) which also coincides with the fundamental resonance wavelength of the high energy electron beam (superscript $f$ ). The dimensionless variables are $\bar{x}=k_{w} x, \bar{y}=k_{w} y, \bar{z}=k_{w} z, \bar{t}=c k_{w} t, \bar{\omega}=\omega / c k_{w}$, $\bar{k}=k / k_{w}, \quad \bar{w}_{h}=k_{w} w_{h}, \quad \bar{X}_{h}=X_{h} / k_{w}, \quad \bar{Y}_{h}=Y_{h} / k_{w}$, $\delta a_{l, n, h}^{(i)}=e \delta A_{l, n, h}^{(i)} / m c^{2}, \bar{s}_{l, n, h}^{(i)}=s_{l, n, h}^{(i)} / k_{w}$, and

$$
\begin{aligned}
\bar{K}_{l, n, h} & =K_{l, n, h} / k_{w} \\
& =(l+n+1)\left(\frac{\alpha_{h}}{\bar{w}_{h}} \frac{d \bar{w}_{h}}{d \bar{z}}-\frac{d \alpha_{h}}{2 d \bar{z}}-\frac{1+\alpha_{h}^{2}}{h \bar{k} \bar{w}_{h}^{2}}\right) .
\end{aligned}
$$

The source terms are

$$
\begin{aligned}
\bar{s}_{l, n, h}^{s}= & \left(\begin{array}{c}
\bar{s}_{l, n, h}^{s,(1)} \\
\bar{s}_{l, n, h}^{s,(2)}
\end{array}\right)=\frac{2 \bar{\omega}_{b, s}^{2}}{h \bar{k}} \frac{F_{l, n}}{\bar{w}_{h}^{2}} \bar{w}_{h}^{2}(0) \\
& \times\left\langle\frac{u_{x}^{s}}{\left|u_{z}^{s}\right|} e_{l, n, h}\left(\bar{x}^{s}, \bar{y}^{s}\right)\left(\begin{array}{c}
-\sin \varphi_{h}^{s} \\
+\cos \varphi_{h}^{s}
\end{array}\right)\right\rangle, \\
\bar{s}_{l, n, 1}^{f}= & \left(\begin{array}{c}
\bar{s}_{l, n, 1}^{f,(1)} \\
\bar{s}_{l, n, 1}^{f,(2)}
\end{array}\right)=\frac{2 \bar{\omega}_{b, f}^{2}}{h^{\prime} \bar{k}} \frac{F_{l, n}}{\bar{w}_{h^{\prime}}^{2}} \bar{w}_{h^{\prime}}^{2}(0) \\
& \times\left\langle\frac{u_{x}^{f}}{\left|u_{z}^{f}\right|} e_{l, n, h^{\prime}}\left(\bar{x}^{f}, \bar{y}^{f}\right)\left(\begin{array}{c}
-\sin \varphi_{1}^{f} \\
+\cos \varphi_{1}^{f}
\end{array}\right)\right\rangle,
\end{aligned}
$$

where $F_{l, n}=\left(2^{l+n} l ! n !\right)^{-1}, \quad \omega_{b, i}^{2}=4 \pi e^{2} n_{b, i} / m, \quad \bar{\omega}_{b, i}^{2}=$ $\omega_{b, i}^{2} / c k_{w}$, and

$$
\begin{gathered}
\varphi_{h}^{s}=\psi_{h}^{s}+\frac{\alpha_{h}\left\lfloor\left(\bar{x}^{s}\right)^{2}+\left(\bar{y}^{s}\right)^{2}\right\rfloor}{\bar{w}_{h}^{2}} \quad h=1,3,5, \ldots, h^{\prime}, \\
\varphi_{1}^{f}=\psi_{1}^{f}+\frac{\alpha_{h^{\prime}}\left\lfloor\left(\bar{x}^{f}\right)^{2}+\left(\bar{y}^{f}\right)^{2}\right\rfloor}{\bar{w}_{h^{\prime}}^{2}} .
\end{gathered}
$$

In obtaining Eq. (8) a symmetry argument is used that electrons that enter the interaction region at time intervals equal to $2 \pi N / \omega$, with $N$ an integer, will have identical trajectories. This is used to exchange the integrals on $t, x$, and $y$ with the integrals on $t_{0}, x_{0}$, and $y_{0}$ in the current [31,32]. Moreover, the recurrence relation of Hermite polynomial together with the assumption that the direct modemode coupling terms can be neglected [30] are also used to find the dynamical equation (8).

It is assumed that the lowest order mode is dominant; therefore, the diffraction of each harmonic component of the radiation field, which is described by the SDE method subject to the interaction with electron beams, is given by [30]

$$
\begin{gathered}
\frac{1}{\bar{w}_{h}} \frac{d \bar{w}_{h}}{d \bar{z}}=\frac{2 \alpha_{h}}{h \bar{k} \bar{w}_{h}^{2}}-\bar{Y}_{h}, \\
\frac{d \alpha_{h}}{2 d \bar{z}}=\frac{1+\alpha_{h}^{2}}{h \bar{k} \bar{w}_{h}^{2}}-\left(\bar{X}_{h}+\alpha_{h} \bar{Y}_{h}\right),
\end{gathered}
$$

where $\bar{X}_{h}$ and $\bar{Y}_{h}$ are given in terms of the source terms by

$$
\begin{gathered}
\bar{X}_{h}=2 \frac{\left(\bar{s}_{0,2, h}^{s,(2)}+\bar{s}_{2,0, h}^{s,(2)}\right) \delta a_{0,0, h}^{(1)}-\left(\bar{s}_{0,2, h}^{s,(1)}+\bar{s}_{2,0, h}^{s,(1)}\right) \delta a_{0,0, h}^{(2)}}{\delta a_{0,0, h}^{(1) 2}+\delta a_{0,0, h}^{(2) 2}}+2 \delta_{h, h^{\prime}} \frac{\left(\bar{s}_{0,2,1}^{f,(2)}+\bar{s}_{2,0,1}^{f,(2)}\right) \delta a_{0,0, h^{\prime}}^{(1)}-\left(\bar{s}_{0,2,1}^{f,(1)}+\bar{s}_{2,0,1}^{f,(1)}\right) \delta a_{0,0, h^{\prime}}^{(2)},}{\delta a_{0,0, h^{\prime}}^{(1) 2}+\delta a_{0,0, h^{\prime}}^{(2) 2}} \\
\bar{Y}_{h}=-2 \frac{\left(\bar{s}_{0,2, h}^{s,(1)}+\bar{s}_{2,0, h}^{s,(1)}\right) \delta a_{0,0, h}^{(1)}+\left(\bar{s}_{0,2, h}^{s,(2)}+\bar{s}_{2,0, h}^{s,(2)}\right) \delta a_{0,0, h}^{(2)}}{\delta a_{0,0, h}^{(1) 2}+\delta a_{0,0, h}^{(2) 2}}-2 \delta_{h, h^{\prime}} \frac{\left(\bar{s}_{0,2,1}^{f,(1)}+\bar{s}_{2,0,1}^{f,(1)}\right) \delta a_{0,0, h^{\prime}}^{(1)}+\left(\bar{s}_{0,2,1}^{f,(2)}+\bar{s}_{2,0,1}^{f,(2)}\right) \delta a_{0,0, h^{\prime}}^{(2)}}{\delta a_{0,0, h^{\prime}}^{(1) 2}+\delta a_{0,0, h^{\prime}}^{(2) 2}} .
\end{gathered}
$$

The relativistic 3D Lorentz force equation of motion for the electrons of the slow (superscript $s$ ) and fast (superscript $f$ ) beams are given, respectively, by 


$$
\begin{gathered}
\frac{d \mathbf{u}^{s}}{d \bar{z}}=-\left\{\frac{1}{u_{z}^{s}}\left(1+\mathbf{u}^{s} \cdot \mathbf{u}^{s}\right)^{1 / 2} \sum_{h=1}^{h^{\prime}} \delta \mathbf{e}_{h}\left(\bar{x}^{s}, \bar{y}^{s}, \bar{z}^{s}\right)+\frac{\mathbf{u}^{s}}{u_{z}^{s}}\left[\mathbf{b}_{w}\left(\bar{x}^{s}, \bar{y}^{s}, \bar{z}^{s}\right)+\sum_{h=1}^{h^{\prime}} \delta \mathbf{b}_{h}\left(\bar{x}^{s}, \bar{y}^{s}, \bar{z}^{s}\right)\right]\right\}, \\
\frac{d \mathbf{u}^{f}}{d \bar{z}}=-\left\{\frac{1}{u_{z}^{f}}\left(1+\mathbf{u}^{f} \cdot \mathbf{u}^{f}\right)^{1 / 2} \delta \mathbf{e}_{h^{\prime}}\left(\bar{x}^{f}, \bar{y}^{f}, \bar{z}^{f}\right)+\frac{\mathbf{u}^{f}}{u_{z}^{f}}\left[\mathbf{b}_{w}\left(\bar{x}^{f}, \bar{y}^{f}, \bar{z}^{f}\right)+\delta \mathbf{b}_{h^{\prime}}\left(\bar{x}^{f}, \bar{y}^{f}, \bar{z}^{f}\right)\right]\right\},
\end{gathered}
$$

where $\delta \mathbf{b}_{h}=\bar{\nabla} \times \mathbf{a}_{r, h}, \quad \delta \mathbf{e}_{h}=-\partial \mathbf{a}_{h} / \partial \bar{t}, \quad \mathbf{u}^{i}=\gamma^{i} \beta^{i}$, $\mathbf{b}_{w}=e B_{w} /\left(m c^{2} k_{w}\right)$, and

$$
\begin{gathered}
\frac{d \bar{x}^{i}}{d \bar{z}}=\frac{u_{x}^{i}}{u_{z}^{i}} \quad i=s, f, \\
\frac{d \bar{y}^{i}}{d \bar{z}}=\frac{u_{y}^{i}}{u_{z}^{i}} \quad i=s, f, \\
\frac{d \psi_{h}^{s}}{d \bar{z}}=h \bar{k}-h \bar{\omega} \frac{\left(1+u_{x}^{s 2}+u_{y}^{s 2}+u_{z}^{s 2}\right)^{1 / 2}}{u_{z}^{s}} \\
h=1,3,5, \ldots, h^{\prime}, \\
\frac{d \psi_{1}^{f}}{d \bar{z}}=h^{\prime} \bar{k}-h^{\prime} \bar{\omega} \frac{\left(1+u_{x}^{f 2}+u_{y}^{f 2}+u_{z}^{f 2}\right)^{1 / 2}}{u_{z}^{f}} .
\end{gathered}
$$

The averaging operator is defined by

$$
\begin{aligned}
\langle(\cdots)\rangle & =\frac{\beta_{z 0}^{i}}{\pi \bar{w}_{h}^{2}(0)} \int d \bar{x}_{0}^{i} d \bar{y}_{0}^{i} \frac{d \psi_{0}^{i}}{2 \pi} \sigma_{\|}^{i}\left(\psi_{0}^{i}\right) \sigma_{\perp}^{i}\left(\bar{x}_{0}^{i}, \bar{y}_{0}^{i}\right)(\cdots) \\
i & =s, f .
\end{aligned}
$$

The initial distributions are assumed to be subject to the normalized conditions

$$
\begin{gathered}
\iint_{\pi\left(\bar{R}_{b}^{i}\right)^{2}} d \bar{x}_{0}^{i} d \bar{y}_{0}^{i} \sigma_{\perp}^{i}\left(\bar{x}_{0}^{i}, \bar{y}_{0}^{i}\right)=\pi\left(\bar{R}_{b}^{i}\right)^{2} \quad i=s, f, \\
\int_{0}^{+2 \pi} \sigma_{\|}^{i}\left(\psi_{0}^{i}\right) d \psi_{0}^{i}=2 \pi \quad i=s, f .
\end{gathered}
$$

Equation (23) is an average over a beamlet of electrons which cross the entry plane within one wave period.

\section{NUMERICAL ANALYSIS}

Equations (8), (13), and (14) together with Eqs. (17)(22) form a set of

$$
6 N_{p 1}+6 N_{p 2}+\sum_{h=1}^{N_{h}} 2\left(M_{h}+1\right),
$$

self-consistent first-order nonlinear differential equations, where $N_{p 1}$ stands for the number of macroparticles in the slow electron beam, $N_{p 2}$ is the number of macroparticles for the fast electron beam, and $N_{h}$ is the number of wavelengths in the system. For example, if the fundamental resonance and its harmonic up-conversion at third harmonic are considered then we will have $N_{h}=2$ or if the fundamental resonance and its fifth harmonic are considered $N_{h}$ will be 3 . Furthermore, $M_{h}$ is the number of modes which is used for each radiation wavelength. These equations may be solved numerically using the fourth-order Runge-Kutta algorithm subject to the appropriate initial conditions. The quantities that are averaged in the dynamical equations are functions of the instantaneous values of the momenta and phases, which are implicit functions of the initial phases of the electrons [32]. It is the initial phase which appears in the averaging operator and represents a convenient method of discretizing the electron beams based upon their initial phase relative to the wave. The number of Gauss-Hermite modes that are needed in the code depends on each particular example. Diffraction over the Rayleigh length is opposed by optical guiding due to the beam, and the balance depends on the Rayleigh length, the growth rate, and the evolution of the beam envelope. Therefore, the specific number of modes used in each case is determined by an empirical procedure in which successive simulation runs are made with an increasing number of modes until convergence of the saturation power and saturation length do not change with the number of modes.

An electron beam with an energy of $219.5 \mathrm{MeV}$, a current of $150 \mathrm{~A}$, and an initial radius of $0.02 \mathrm{~cm}$ is chosen as a low energy electron beam. An electron beam with an energy of $380.185 \mathrm{MeV}$, a current of $300 \mathrm{~A}$, and an initial radius of $0.02 \mathrm{~cm}$ is exploited as a fast electron beam. The wiggler field amplitude is $B_{w}=10.06 \mathrm{kG}$, period is $\lambda_{w}=$ $3.3 \mathrm{~cm}$, and an entry taper region is $N_{w}=10$ wiggler period in length. The initial condition of the radiation fields is chosen such that the fundamental wavelength is seeded with a $10 \mathrm{~W}$ of optical power which is also assumed to be totally in the lowest mode of fundamental resonance. The harmonic has started from zero initial power. The initial radiation waists are $0.05 \mathrm{~cm}$ and the initial alpha parameters are chosen to be zero. For the chosen parameters of the electron beam and wiggler magnetic field, the 1D resonance formula yields a wavelength of $496.1 \mathrm{~nm}$. Because of three-dimensional effects, fundamental resonance takes place at $500.5 \mathrm{~nm}$ and the third harmonic is at $166.84 \mathrm{~nm}$. The averages in the dynamical equations for the wave modes are performed by means of an $N$ th-order Gaussian quadrature technique in each of the variables. The initial state of electron beams is chosen to model the injection of a monoenergetic and axisymmetric electron beams with the flattop density profiles, i.e., $\sigma_{\perp}=1$. For unbunched electron beam the particles are uniformly distributed in phase. On the other hand, for the prebunched case, the beam is 
modeled by the following distribution in initial phase [33]:

$$
\sigma\left(\psi_{0}\right)= \begin{cases}\left(4 \pi / \psi_{\text {width }}\right) \sin ^{2}\left(\pi \psi_{0} / \psi_{\text {width }}\right) & 0 \leq \psi_{0} \leq \psi_{\text {width }}, \\ 0 & \text { otherwise }\end{cases}
$$

where $\psi_{0}=-\omega t_{0}$. The electron positions are chosen by means of the Gaussian algorithm within the ranges $0 \leq$ $\psi_{0} \leq 2 \pi, 0 \leq \theta_{0} \leq 2 \pi$, and $0 \leq r_{0} \leq R_{b}$, where $\theta_{0}$ is the polar coordinate. In the absence of an energy spread, $p_{z 0}=$ $m c\left(\gamma_{0}^{2}-1\right)^{1 / 2}$, where $\gamma_{0}$ is the relativistic factor corresponding to the total beam energy. It is important to recognize, however, that the subsequent evolution of the beam is integrated self-consistently, and the beam may bunch in axial phases as well as develop both energy and pitch angle spreads due to the nature of the interaction. For each wavelength in the system, 36 modes are used with a total of 4096 particles for each electron beam; therefore the total number of particles is 8192 . The code is run on the AMD Phenom ${ }^{\mathrm{TM}} \mathrm{X} 3$ Triple Core processor. In order to check the validity of our code parameters of Ref. [30] for a single beam is used and identical results are obtained.

In two-beam FEL, the external seed for low energy electron beam is used. Therefore, it is irrelevant to consider the effect of shot noise for fundamental resonance of the low energy electron beam. The third harmonic in the system is started from the shot noise but in the nonlinear regime the harmonic bunching due to the fundamental resonance creates seed for it. Therefore, the effect of shot noise is not important which is also stated in Ref. [12]. It is worth mentioning that the effect of slippage must be considered for a system in which radiation pulse moves out of the electron pulse, considerably. In our system, the wiggler length is $13.4 \mathrm{~m}$. After each wiggler period, i.e., each $3.3 \mathrm{~cm}$, radiation slips one wavelength over the electron pulse. Therefore, for fundamental resonance the slippage length is $200 \mu \mathrm{m}$. If the length of the electron beam is about $2 \mathrm{~mm}$, only one-tenth of the radiation pulse can escape the electron beam. Therefore, slippage is negligible and the steady-state simulation can be applied.

The power of the fundamental resonance and harmonic up-conversion at the third harmonic for water bag distributions of entry times are plotted in Fig. 1(a) as a function of the distance through the system. In contrast to the nonlinear harmonic generation [30,34], the intensity of the shorter wavelength is larger than the intensity of fundamental wavelength [12]. This means that by seeding the fundamental resonance of the lower energy beam, energy will be up converted to the higher frequency third harmonic leading to higher power. The fundamental resonance, in Fig. 1(a), has three distinct regime, i.e., small signal regime that ends at around $z \approx 2 \mathrm{~m}$, exponential growth, and saturation at $z=10.76 \mathrm{~m}$. These three regimes for the third harmonic, on the other hand, are not distinctly well separated and saturation is at $z=13.48 \mathrm{~m}$. The reason is that the third harmonic is driven by both beams and it is the
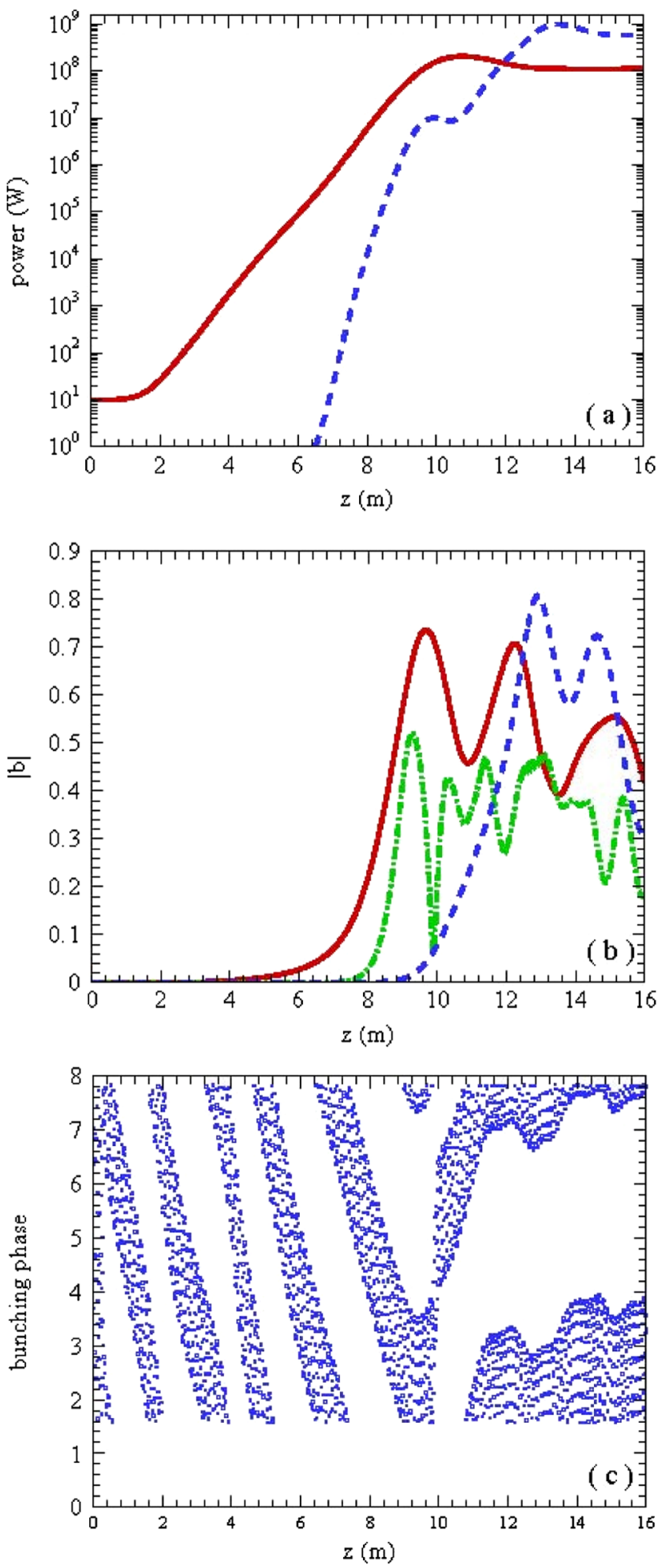

FIG. 1. (Color) (a) Evolution of the power for fundamental resonance (solid line) and its harmonic up-conversion at third harmonic (dashed line). (b) Bunching parameter for fundamental resonance wavelength (solid line), its third harmonic (dashdotted line) for low energy electron beam, and fundamental resonance of high energy electron beam (dashed line). (c) Phase of bunching parameter for third harmonic of low energy electron beam with longitudinal coordinate. 
superposition of two different types of amplifying radiation; the fundamental resonance of the high energy beam and the third harmonic of the low energy beam.

Note that the points of saturation vary for wavelength, as is evident in Fig. 1(a) as well as in Fig. 2 in more detail. Saturated power of the fundamental frequency is $2.05 \times$ $10^{8}(\mathrm{~W})$ and third harmonic power saturates at $9.86 \times$ $10^{8}(\mathrm{~W})$. Bunching parameter of fundamental resonance and its third harmonic for the low energy electron beam together with the bunching parameter of fundamental resonance of high energy electron beam are plotted in Fig. 1(b). In Fig. 1(c), the phase of bunching parameter of the third harmonic of low energy electron beam is plotted. The first peak of bunching parameter for fundamental resonance wavelength of low energy electron beam that occurs at $z=$ $9.67 \mathrm{~m}$ is 0.73 . For the third harmonic of low energy electron beam, the first peak of bunching parameter is 0.52 that takes place at $z=9.27 \mathrm{~m}$. The saturated value of bunching parameter for fundamental resonance of the high energy electron beam is 0.8 that occurs at $z=12.9 \mathrm{~m}$. In Figs. 2(a) and 2(b) evolution of the radiation amplitude in the transverse plane is shown as a function of $z$ for the fundamental mode and the third harmonic, respectively. All transverse profiles are normalized to the peak intensity of 1 . Therefore these figures do not show the amplification of the radiation. It can be seen that the amplitude profile of the radiation in the transverse plane becomes narrower as the radiation travels along the wiggler. Narrowing of the transverse intensity profile is evident as the radiation propagates toward the point of saturation and this mode narrowing is greater for the third harmonic. Although the approximate position of saturation points can be seen from the intensity profile of Fig. 1, its more precise position can be inferred from the point where mode narrowing stops and the intensity profile widens. This is because radiation waist begins to grow at saturation since the socalled gain guiding is no longer effective. To determine the exact position of saturation, on this basis, an extensive numerical investigation is necessary with this code to read out many more modal maps in $z$. Before the saturation point, strong Gaussian profiles with gentle ripples on their outskirts indicate that $\mathrm{TEM}_{00}$ is the dominant mode in this region. However, additional modes tend to grow, beyond the saturation point.

In Fig. 3, the spot size or the radiation waist of fundamental resonance and third harmonic up converted radiation are compared. The radiation spot size for fundamental resonance is observed to expand, from its initial size of $w_{1}(0)=0.05 \mathrm{~cm}$, during the initial stage of interaction as predicted by vacuum diffraction. However, the radiation spot size experiences rapid focusing at the onset of exponential growth, and undergoes a few oscillations during this phase before expanding rapidly as saturation occurs. The radiation spot size for the third harmonic behaves to some extent differently, compared to the fundamental
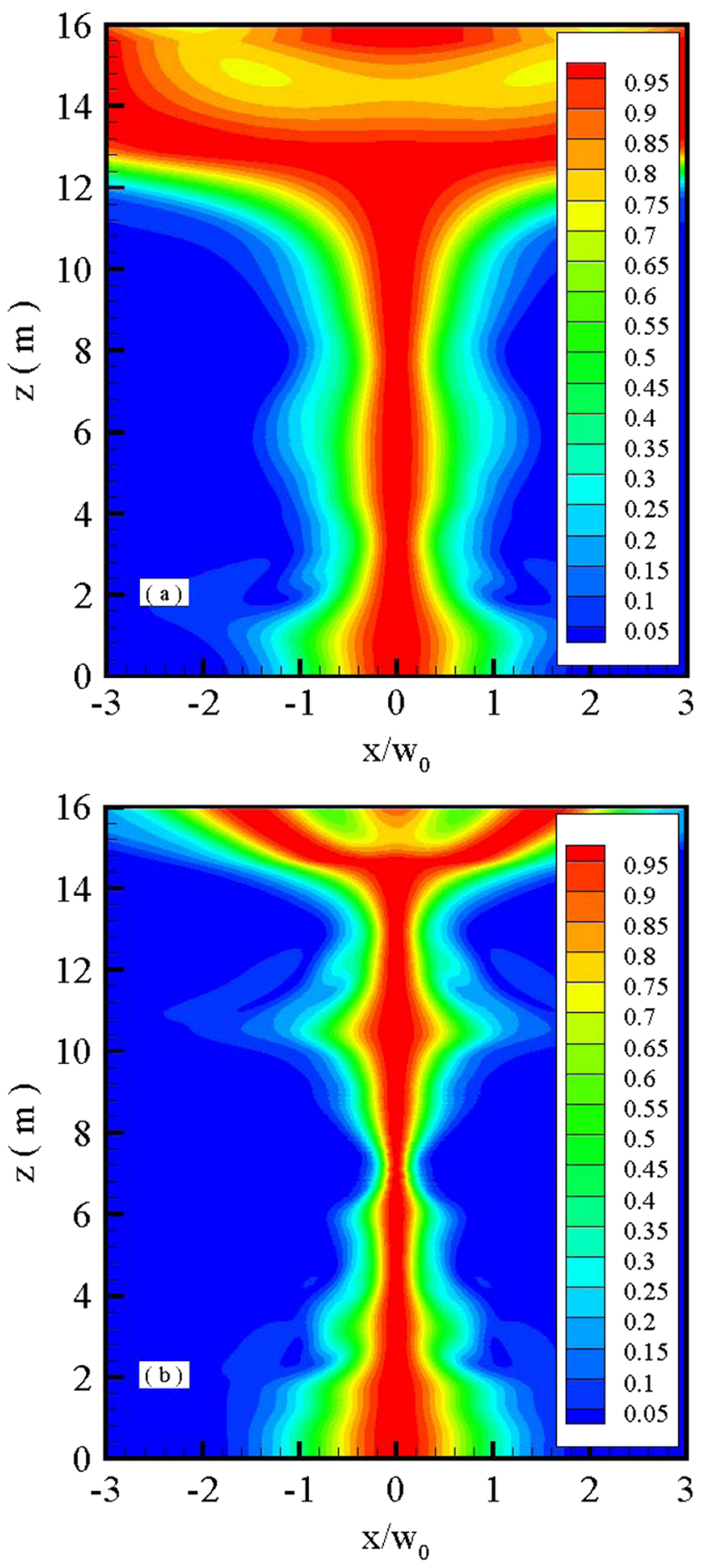

FIG. 2. (Color) Transverse intensity profile of fundamental resonance wavelength (a) and third harmonic wavelength (b) in the $x$ direction for $y=0$.

resonance, but is always smaller, especially at saturation which becomes very small. In the single beam FEL, the spot size of the third harmonic is larger than that of the fundamental resonance in the region of the exponential growth but remains small in the small signal regime and beyond saturation [30]. 




FIG. 3. (Color) Evolution of radiation spot size for fundamental wavelength (solid line) and its harmonic up-conversion at third harmonic (dashed line) with longitudinal coordinate.

Although the presence of higher order modes are evident in Fig. 2 beyond the saturation point, they only constitute about $20 \%$ of the total power and the other $80 \%$ is concentrated in the lowest order $\mathrm{TEM}_{00}$ mode. These points are illustrated in Fig. 4 where the evolution of the total power, due to all modes, and that of the lowest order $\mathrm{TEM}_{00}$ mode for the fundamental resonance and for the third harmonic are shown. It can be seen that the lowest order mode is dominant. Therefore our assumption that the lowest order mode is dominant is justified. Among the 35 other modes, $\mathrm{TEM}_{02}, \mathrm{TEM}_{04}, \mathrm{TEM}_{20}, \mathrm{TEM}_{22}, \mathrm{TEM}_{24}$, $\mathrm{TEM}_{40}, \mathrm{TEM}_{42}$, and $\mathrm{TEM}_{44}$ modes have a noticeable contribution and the rest are negligible.

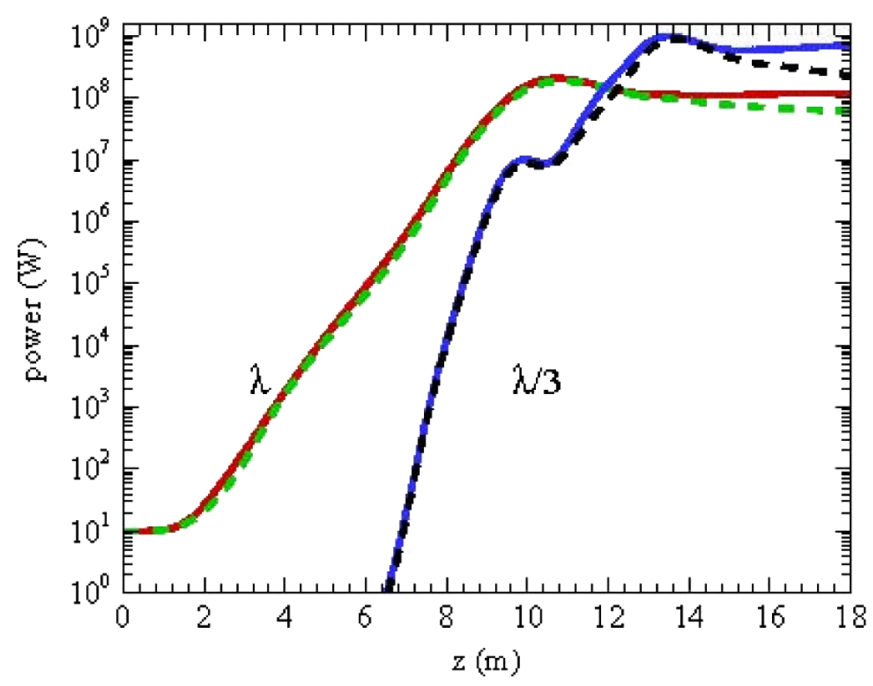

FIG. 4. (Color) Evolution of the total power (solid line) and that of the $\mathrm{TEM}_{00}$ mode (dashed line) for the fundamental resonance and the third harmonic.

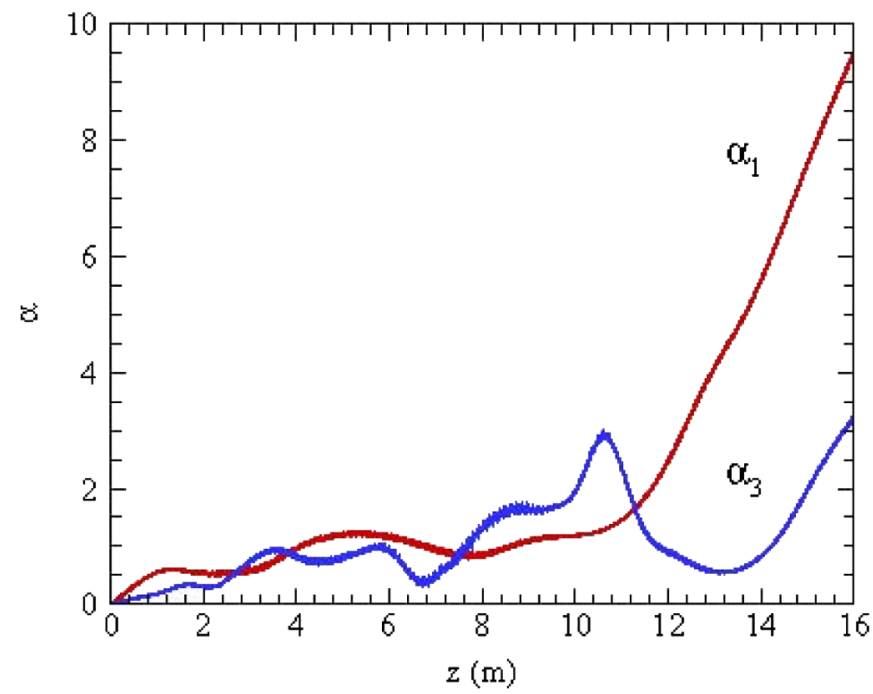

FIG. 5. (Color) Evolution of $\alpha_{1}$ and $\alpha_{3}$ with longitudinal coordinate.

The evolution of $\alpha$, which represents the curvature of the phase front, is shown in Fig. 5. Both fundamental resonance and the third harmonic, which are plane waves at the entrance to the undulator at $z=0$, deviate from plane waves as radiation moves forward and increases abruptly once saturation is passed.

In Fig. 6(a), the effect of sinusoidal distribution in entry times for low energy electron beam on the evolution of radiation power in the system is illustrated. It can be seen that for sinusoidal distribution the evolution of radiation power has three regimes which are over exponential, exponential, and saturation. By decreasing the prebunching width, the exponential regime disappears and saturation length decreases dramatically. For shorter wavelengths more complicated means is required for the production of a prebunched electron beam and also the degree of prebunching is reduced. For these reasons, the effect of prebunching is only considered for the low energy electron beam. For sinusoidal distribution of the low energy electron beam with $\psi_{\text {width }}=2 \pi$, saturated power of fundamental resonance wavelength is $4.2 \times 10^{8} \mathrm{~W}$ which takes place at $z=3.57 \mathrm{~m}$ and saturated power of the third harmonic is $9.9 \times 10^{8} \mathrm{~W}$ and saturation length is $6.51 \mathrm{~m}$. Because of the significant reduction of saturation length the effect of slippage, in this case, is less important. The electron-beam pulse in this case can be reduced to $0.98 \mathrm{~mm}$. The bunching parameter of fundamental resonance and its third harmonic for the low energy electron beam together with the bunching parameter of fundamental resonance of the high energy electron beam are plotted in Fig. 6(b). In Fig. 6(c), the phase of bunching parameter of third harmonic of low energy electron beam is plotted. The first peak of bunching parameter for fundamental resonance wavelength of low energy electron beam that occurs at $z=3.12 \mathrm{~m}$ is 0.81 . For the third harmonic of the low 

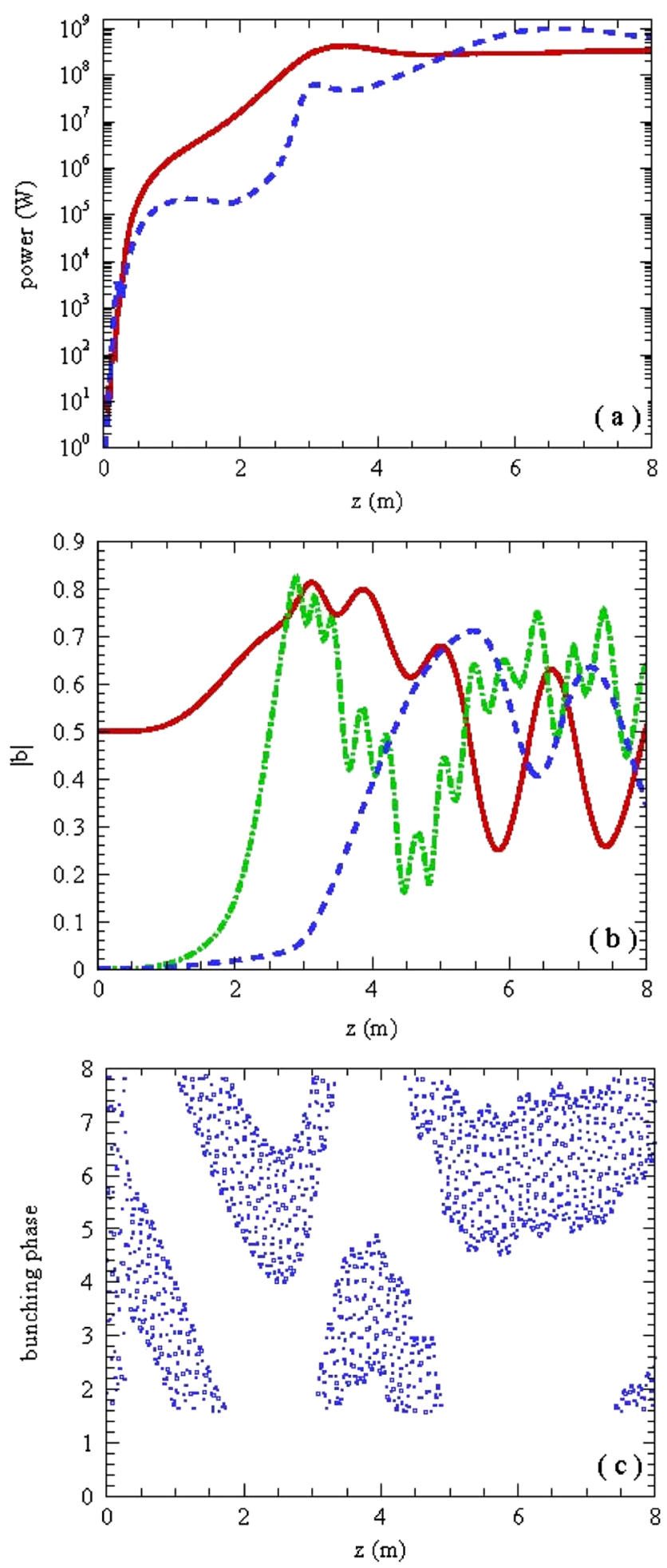

FIG. 6. (Color) (a) Evolution of the power for fundamental resonance (solid line) and its harmonic up-conversion at third harmonic (dashed line). (b) Bunching parameter for fundamental resonance wavelength (solid line), its third harmonic (dashdotted line) for low energy electron beam, and fundamental resonance of high energy electron beam (dashed line). (c) Phase of bunching parameter for third harmonic of low energy electron beam with longitudinal coordinate when low energy electron beam is prebunched with sinusoidal distribution.

energy electron beam, the first peak of bunching parameter is 0.82 that takes place at $z=2.89 \mathrm{~m}$. The saturated value of the bunching parameter for fundamental resonance of the high energy electron beam is 0.71 that occurs at $z=$ $5.46 \mathrm{~m}$. For the prebunched electron beam with $\psi_{\text {width }}=$ $2 \pi$ the evolution of radiation waist and radiation amplitude in the transverse plane are illustrated in Figs. 7 and 8.

For the second example, an electron beam with an energy of $470 \mathrm{MeV}$, a current of $300 \mathrm{~A}$, and an initial radius of $0.1495 \mathrm{~mm}$ is chosen as a low energy electron beam. An electron beam with an energy of $799 \mathrm{MeV}$, a current of $600 \mathrm{~A}$, and an initial radius of $0.1495 \mathrm{~mm}$ is exploited as a fast electron beam. The wiggler parameters are as before. The fundamental resonance wavelength is seeded with a $10 \mathrm{~W}$ of radiation which is also assumed to be totally in the lowest mode of fundamental resonance. The harmonic wavelength starts from zero initial power. The initial radiation waists are $0.37 \mathrm{~mm}$ and the initial alpha parameters are chosen to be zero. For the chosen parameters of the electron beam and wiggler magnetic field the fundamental resonance takes place at $107.5 \mathrm{~nm}$ and the third harmonic is at $35.8 \mathrm{~nm}$. The variation of power for fundamental resonance wavelength versus wavelength at $z=10 \mathrm{~m}$ is plotted in Fig. 9 .

The power of the fundamental resonance and harmonic up-conversion at the third harmonic for water bag distributions of entry times are plotted in Fig. 10(a) as a function of the distance through the system. The saturated power of the fundamental frequency is $5.88 \times 10^{8}(\mathrm{~W})$ which occurs at $z=11.14 \mathrm{~m}$ and the third harmonic power saturates at $1.81 \times 10^{9}(\mathrm{~W})$ which takes place at $z=13.63 \mathrm{~m}$. The bunching parameter of fundamental resonance and its third harmonic for the low energy electron beam together with a bunching parameter of fundamental resonance of the

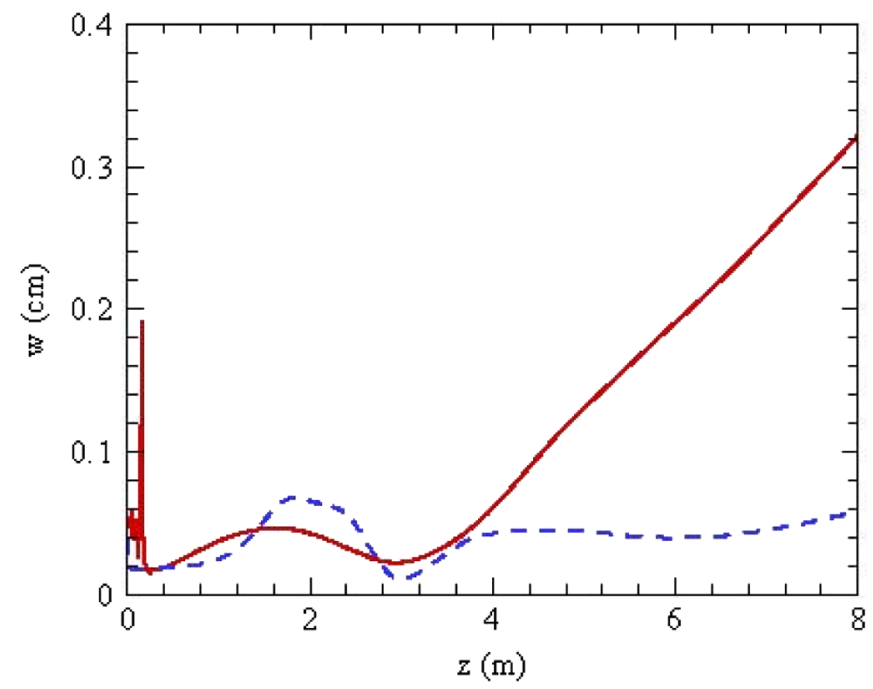

FIG. 7. (Color) Evolution of radiation spot size for fundamental wavelength (solid line) and its harmonic up-conversion at third harmonic (dashed line) when low energy electron beam is prebunched with sinusoidal distribution. 

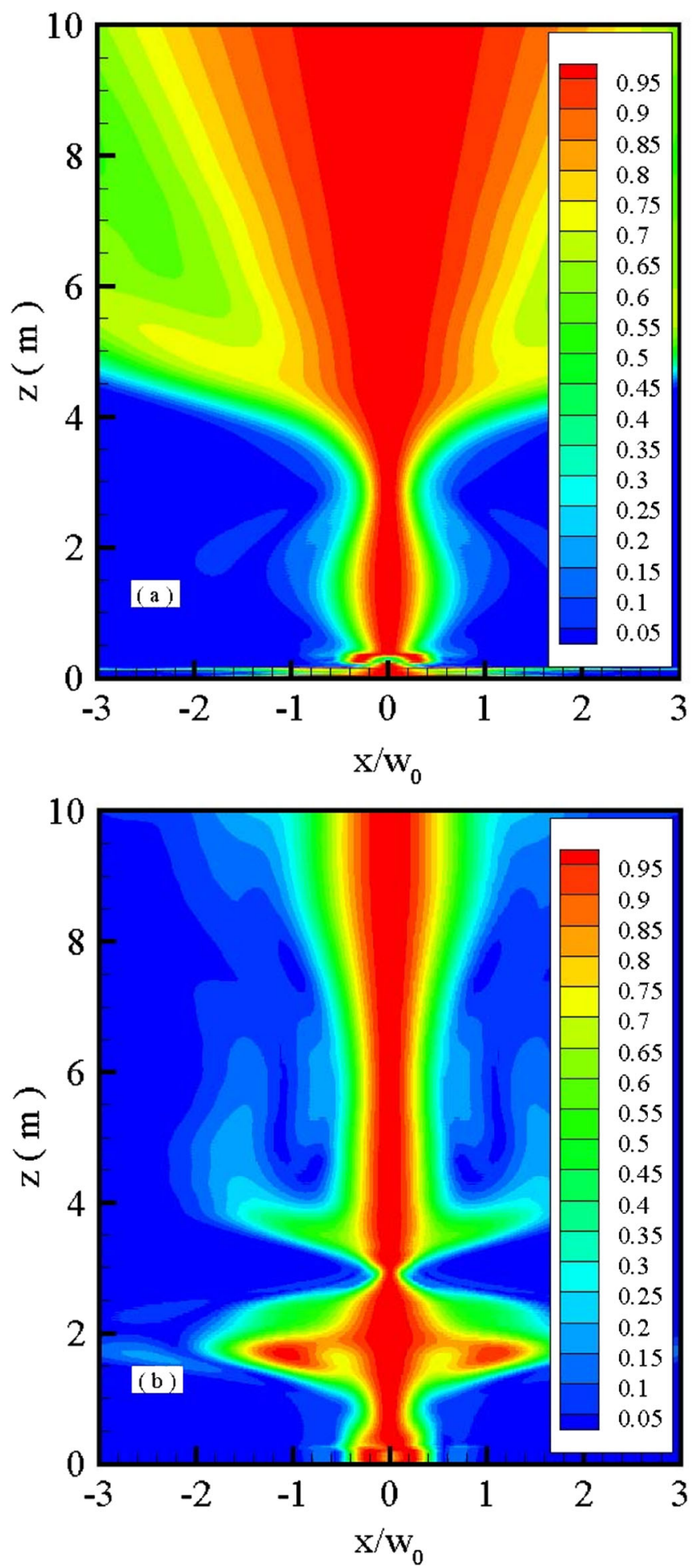

FIG. 8. (Color) Transverse intensity profile of fundamental resonance wavelength (a) and third harmonic wavelength (b) in the $x$ direction for $y=0$ when low energy electron beam is prebunched with sinusoidal distribution.

high energy electron beam are plotted in Fig. 10(b). In Fig. 10(c), the phase of the bunching parameter of the third harmonic of the low energy electron beam is plotted. The

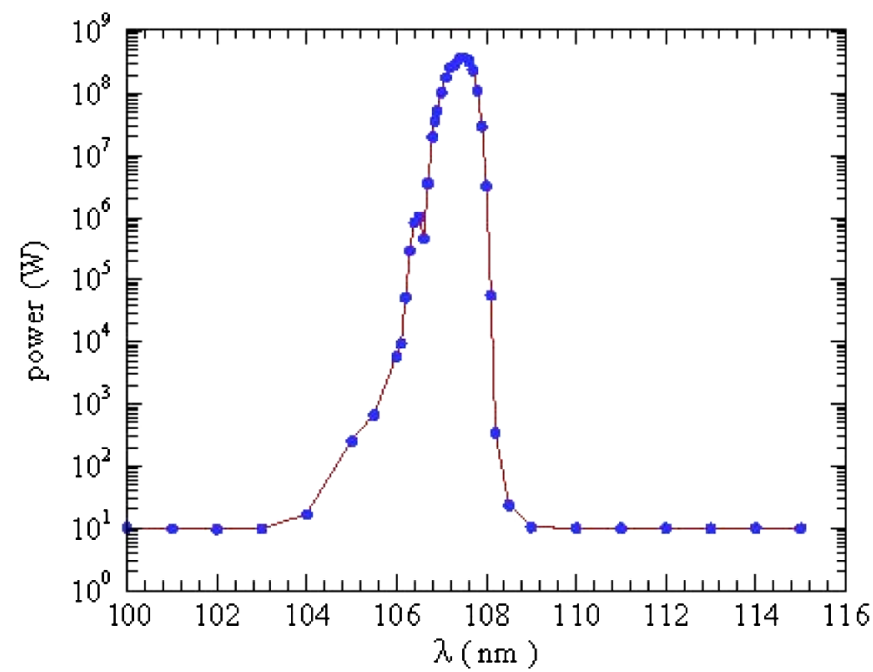

FIG. 9. (Color) Variation of power for fundamental resonance wavelength for low energy electron beam versus wavelength at $z=10 \mathrm{~m}$.

first peak of the bunching parameter for fundamental resonance wavelength of the low energy electron beam that occurs at $z=10.15 \mathrm{~m}$ is 0.77 . For the third harmonic of the low energy electron beam, the first peak of the bunching parameter is 0.55 that takes place at $z=9.62 \mathrm{~m}$. The saturated value of the bunching parameter for fundamental resonance of the high energy electron beam is 0.73 that occurs at $z=12.41 \mathrm{~m}$. In Fig. 11 evolution of radiation waists is presented. For comparison, the evolution of radiation waist for the fundamental resonance wavelength in the absence of electron beams is also plotted. The effect of gain guiding due to the electron beam is evident.

The transformation matrix for symmetric quadruple triplet or FODO ( $\mathrm{F}$ and D correspond to the focusing and defocusing quadruple magnet, respectively, and $\mathrm{O}$ is for the drift space) lattice that starts at the middle of a focusing quadruple is $[35,36]$

$$
\mathbf{M}_{\mathrm{FODO}}=\mathbf{M}_{1 / 2 Q F} \mathbf{M}_{O} \mathbf{M}_{Q D} \mathbf{M}_{O} \mathbf{M}_{1 / 2 Q F}=\left[\begin{array}{cc}
C & S \\
C^{\prime} & S^{\prime}
\end{array}\right],
$$

where $\mathbf{M}_{1 / 2 Q F}$ is the transformation matrix for quadruple focusing, $\mathbf{M}_{O}$ is for drift space between quadruples, and $\mathbf{M}_{Q D}$ is for quadruple defocusing. In the FODO lattice the matched electron beam has the periodicity of the lattice. Therefore, the beam envelope must satisfy

$$
r_{b}\left(z+L_{p}\right)=r_{b}(z)
$$

where $L_{p}$ is the lattice period, i.e.,

$$
\kappa\left(z+L_{p}\right)=\kappa(z),
$$

where $\kappa$ is the lattice function. The value of $\kappa$ in terms of magnetic field gradient and electron beam energy can be written as [37] 

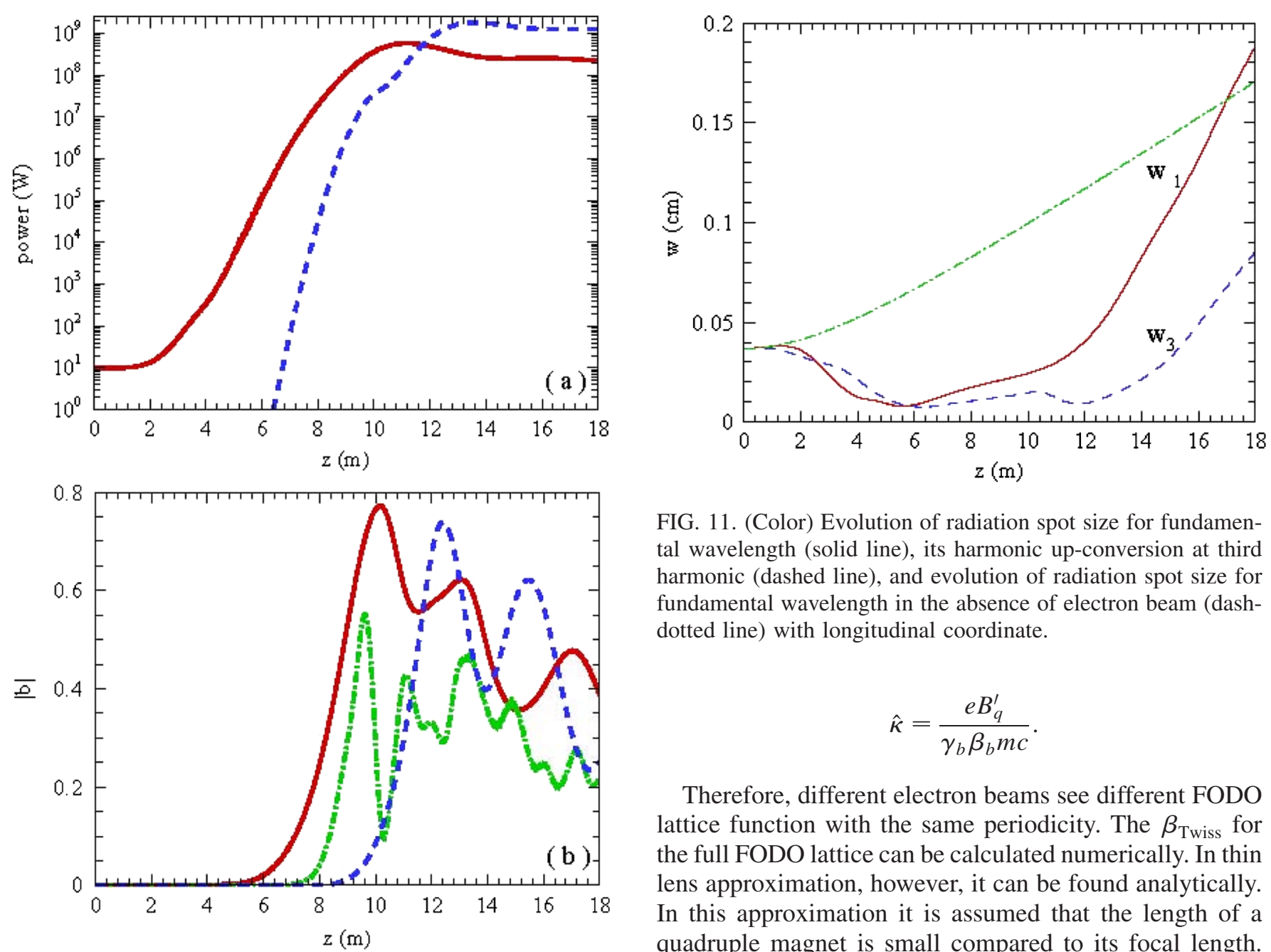

FIG. 11. (Color) Evolution of radiation spot size for fundamental wavelength (solid line), its harmonic up-conversion at third harmonic (dashed line), and evolution of radiation spot size for fundamental wavelength in the absence of electron beam (dashdotted line) with longitudinal coordinate.

$$
\hat{\kappa}=\frac{e B_{q}^{\prime}}{\gamma_{b} \beta_{b} m c} .
$$

Therefore, different electron beams see different FODO lattice function with the same periodicity. The $\beta_{\text {Twiss }}$ for the full FODO lattice can be calculated numerically. In thin lens approximation, however, it can be found analytically. In this approximation it is assumed that the length of a quadruple magnet is small compared to its focal length. The elements of the transformation matrix of FODO lattice in this approximation reduce to $C=1-2 L^{2} f^{-2}, S=$ $2 L(1+L / f), C^{\prime}=-1 / f^{*}$, and $S^{\prime}=1-2 L^{2} f^{-2}$. Here $f$ is the focal length, $L$ is the half length of the lattice, and $f^{*-1}=2(1-L / f) L / f^{2}$. In this approximation, for $\alpha_{\text {Twiss }}(0)=0$, the ratio of $\beta_{\text {Twiss }}$ for electron beams is

$$
\frac{\beta_{\mathrm{Twiss}}^{f}}{\beta_{\mathrm{Twiss}}^{s}}=\frac{\hat{\kappa}^{s}}{\hat{\kappa}^{f}}
$$

Therefore the ratio of the electron beam radius $(r=$ $\left.\sqrt{\varepsilon \beta_{\text {Twiss }}}\right)$ can be written as

$$
\frac{r^{s}}{r^{f}}=\sqrt{\frac{\varepsilon_{n}^{s}}{\varepsilon_{n}^{f}}},
$$

where $\varepsilon_{n}$ with superscript $s(f)$ is the normalized emittance of slow (fast) electron beam. Therefore two different electron beams can be matched to one FODO lattice. As shown in [38], in a single undulator, the matched radius of electron beam is proportional to $\sqrt{\varepsilon_{n}}$. If the normalized emittance for electron beams is chosen to be $3 \pi \mathrm{mm} \mathrm{mrad}$, the matched radius for electron beams is $0.1495 \mathrm{~mm}$. Therefore two electron beams with different energy not 
only match to the same wiggler but also can be matched to the FODO lattice.

\section{CONCLUSION AND SUGGESTION}

A nonaveraged and 3D simulation of harmonic upconversion in a FEL amplifier operating simultaneously with two cold and relativistic electron beams with different energy is presented in the absence of slippage. The variation of radiation waists, curvatures, and amplitudes for fundamental and its harmonic up-conversion are studied. Transverse mode evolution of fundamental and the harmonic up-conversion at the third harmonic are investigated in more detail. The radiation power of harmonic upconversion is larger than that of the fundamental resonance. This is in contrast to the nonlinear harmonic generation. It is also in contrast to the radiation up-conversion method in which the wiggler is filled by plasma [39]. The waist of harmonic up converted radiation is focused to a smaller spot size than that of the fundamental resonance. At the point in which the harmonic up converted radiation saturates the fundamental radiation loses its quality, because it has passed beyond its own saturation point. The effect of prebunching of low energy electron beam on the evolution of radiation in the system is studied. Using low energy prebunched electron beam dramatically reduced saturation length.

The numerical simulation, with the SDE technique in three dimensions, of the two-beam FEL shows that by seeding the lower energy beam considerable seeding of both the radiation field and the electron bunching of the higher energy beam is possible. This proposed seeding method may find application in the FELs in extreme ultraviolet or $\mathrm{X}$-ray domain where direct coherent seeding is not possible.

Extension of this work to study the optical properties of harmonic up converted radiation by using the $M^{2}$ parameter [40] for the two-beam FEL is in progress. The effect of wiggler contouring for efficiency enhancement will be studied in the future.

\section{APPENDIX}

Here, a detailed derivation of Eq. (5) is presented. For simplicity harmonics are not considered. The radiation amplitude, for the fundamental resonance, is

$$
\mathbf{A}_{r}(\mathbf{x}, t)=\frac{1}{2} A_{r}(\mathbf{x}, t) \exp (i k z-i \omega t) \hat{\mathbf{e}}_{x}+\text { c.c. },
$$

where $A_{r}(\mathbf{x}, t)$ is the radiation amplitude that varies slowly with $z$ and $t$, but we do not impose any restriction on $x$ and $y$. Substituting this form of the radiation amplitude in the hyperbolic wave equation,

$$
\begin{aligned}
& \left(\nabla_{\perp}^{2}+\frac{\partial^{2}}{\partial z^{2}}-\frac{1}{c^{2}} \frac{\partial^{2}}{\partial t^{2}}\right)\left[\frac{1}{2} A_{r}(\mathbf{x}, t) \exp (i k z-i \omega t) \hat{\mathbf{e}}_{x}+\text { c.c. }\right] \\
& =-\frac{4 \pi}{c} \mathbf{J},
\end{aligned}
$$

leads to

$$
\begin{gathered}
e^{i(k z-\omega t)} \nabla_{\perp}^{2} \frac{A_{r}}{2}+D_{+} D_{-}\left\{\frac{A_{r}}{2} e^{i(k z-\omega t)}\right\}+e^{-i(k z-\omega t)} \nabla_{\perp}^{2} \frac{A_{r}^{*}}{2} \\
+D_{+} D_{-}\left\{\frac{A_{r}^{*}}{2} e^{-i(k z-\omega t)}\right\}=-\frac{4 \pi}{c} J_{x},
\end{gathered}
$$

where $D_{+}=\partial / \partial z+c^{-1} \partial / \partial t$ and $D_{-}=\partial / \partial z_{-}$ $c^{-1} \partial / \partial t$. Applying $D_{+} D_{-}$gives

$$
D_{+} D_{-}\left\{A_{r} e^{i(k z-\omega t)}\right\}=e^{i(k z-\omega t)}\left(D+2 i k D_{+}\right) A_{r} .
$$

Here, $D$ can be neglected because it is proportional to the second order derivatives of amplitude with respect to $z$ and $t$. Therefore,

$$
D_{+} D_{-}\left\{A_{r} e^{i(k z-\omega t)}\right\} \cong 2 i k e^{i(k z-\omega t)} D_{+} A_{r} .
$$

By using the above relation, Eq. (A2) reduces to

$$
\begin{aligned}
& e^{i(k z-\omega t)}\left[\nabla_{\perp}^{2}+2 i k D_{+}\right] \frac{A_{r}}{2}+e^{-i(k z-\omega t)}\left[\nabla_{\perp}^{2}+2 i k D_{+}\right] \frac{A_{r}^{*}}{2} \\
& =-\frac{4 \pi}{c} J_{x} .
\end{aligned}
$$

Multiplying the above equation by $e^{-i(k z-\omega t)}$, and integrating the resulting equation over one wave period, removes the second term which has rapid oscillation and the parabolic equation is obtained:

$$
\left(\nabla_{\perp}^{2}+2 i k D_{+}\right) A_{r}=-\frac{8 \pi}{c} \frac{\omega}{2 \pi} \int_{0}^{2 \pi / \omega} J_{x} e^{-i(k z-\omega t)} d t .
$$

In the absence of slippage $D_{+}$reduces to $\partial / \partial z$ and Eq. (5) will be obtained for $h=1$.

[1] K. J. Gaffney and H.N. Chapman, Science 316, 1444 (2007).

[2] H. N. Chapman et al., Nature Phys. 2, 839 (2006).

[3] J. Kirz, Nature Phys. 2, 799 (2006).

[4] S. P. Hau-Riege et al., Phys. Rev. Lett. 98, 145502 (2007).

[5] A. P. Mancuso et al., Phys. Rev. Lett. 102, 035502 (2009).

[6] B. McNeil, Nat. Photon. 3, 375 (2009); W. Ackermann et al., Nat. Photon. 1, 336 (2007).

[7] A. Singer, I. A. Vartanyants, M. Kuhlmann, S. Duesterer, R. Treusch, and J. Feldhaus, Phys. Rev. Lett. 101, 254801 (2008).

[8] G. Lambert et al., Nature Phys. 4, 296 (2008).

[9] L.H. Yu, M. Babzien, I. Ben-Zvi, L.F. DiMauro, A. Doyuran, W. Graves, E. Johnson, S. Krinsky, R. Malone, I. Pogorelsky, and J. Skaritka, Science 289, 932 (2000).

[10] D. Xiang and G. Stupakov, Phys. Rev. ST Accel. Beams 12, 030702 (2009). 
[11] G. Stupakov, Phys. Rev. Lett. 102, 074801 (2009).

[12] B. W. J. McNeil, G. R. M. Robb, and M. W. Poole, Phys. Rev. E 70, 035501(R) (2004).

[13] G. T. Moore, Nucl. Instrum. Methods Phys. Res., Sect. A 239, 19 (1985).

[14] E. T. Scharlemann, A. M. Sessler, and J. S. Wurtele, Phys. Rev. Lett. 54, 1925 (1985).

[15] E. Hemsing, A. Gover, and J. Rosenzweig, Phys. Rev. A 77, 063830 (2008).

[16] S. G. Biedron, H. P. Freund, S. V. Milton, G. Dattoli, A. Renieri, and P. L. Ottaviani, Nucl. Instrum. Methods Phys. Res., Sect. A 528, 443 (2004).

[17] H. P. Freund, D. Douglas, and P. G. O'Shea, Nucl. Instrum. Methods Phys. Res., Sect. A 507, 373 (2003).

[18] P. Sprangle, A. Ting, and C. M. Tang, Phys. Rev. Lett. 59, 202 (1987).

[19] B. Hafizi, P. Sprangle, and A. Ting, Phys. Rev. A 36, 1739 (1987).

[20] P. Sprangle, A. Ting, and C. M. Tang, Phys. Rev. A 36, 2773 (1987).

[21] P. Sprangle, A. Ting, B. Hafizi, and C. M. Tang, Nucl. Instrum. Methods Phys. Res., Sect. A 272, 536 (1988).

[22] B. Hafizi, P. Sprangle, and J. R. Penano, Nucl. Instrum. Methods Phys. Res., Sect. A 581, 601 (2007).

[23] C. M. Tang and P. Sprangle, IEEE J. Quantum Electron. 21, 970 (1985).

[24] N. M. Kroll, P. L. Morton, and M. N. Rosenbluth, IEEE J. Quantum Electron. 17, 1436 (1981).

[25] S. Reiche, Nucl. Instrum. Methods Phys. Res., Sect. A 429, 243 (1999).
[26] T. M. Tran and J. S. Wurtele, Comput. Phys. Commun. 54, 263 (1989).

[27] T. M. Tran and J. S. Wurtele, Phys. Rep. 195, 1 (1990).

[28] E. L. Saldin, E. A. Schneidmiller, and M. V. Yurkov, Nucl. Instrum. Methods Phys. Res., Sect. A 429, 233 (1999).

[29] W. Fawley, LBID-2141, CBP Tech Note-104, UC-414, 1995.

[30] H.P. Freund, S. G. Biedron, and S. V. Milton, IEEE J. Quantum Electron. 36, 275 (2000).

[31] P. Sprangle, C. M. Tang, and W. M. Manheimer, Phys. Rev. A 21, 302 (1980).

[32] H.P. Freund and J.M. Antonsen, Principle of Free Electron Laser (Chapman and Hall, London, 1996), Chap. 5.

[33] H. P. Freund, P. G. O'Shea, and J. Neumann, Nucl. Instrum. Methods Phys. Res., Sect. A 507, 400 (2003).

[34] H. P. Freund, L. Giannessi, and W. H. Miner, Jr., J. Appl. Phys. 104, 123114 (2008).

[35] H. Wiedemann, Particle Accelerator Physics (SpringerVerlag, Berlin, 2007).

[36] M. Reiser, Theory and Design of Charged Particle Beams (Wiley-VCH, Weinheim, 2004).

[37] S. M. Lund and B. Bukh, Phys. Rev. ST Accel. Beams 7, 024801 (2004).

[38] H. P. Freund and P. G. O'Shea, Phys. Rev. Lett. 80, 520 (1998); Nucl. Instrum. Methods Phys. Res., Sect. A 429, 219 (1999).

[39] V. Petrillo and C. Maroli, Phys. Rev. E 62, 8612 (2000).

[40] P. Sprangle, H. P. Freund, B. Hafizi, and J. R. Penano, IEEE J. Quantum Electron. 45, 218 (2009). 